\title{
Several types of well-posedness for generalized vector quasi-equilibrium problems with their relations
}

De-ning $\mathrm{Qu}^{1,2^{*}}$ and Cao-zong Cheng ${ }^{1}$

To Prof. W. Takahashi on the occasion of his 70th birthday

${ }^{*}$ Correspondence:

qudening@126.com

${ }^{1}$ College of Applied Science, Beijing

University of Technology, Beijing,

100124, P.R. China

${ }^{2}$ College of Mathematics, Jilin Normal University, Jilin, 136000 ,

P.R. China

\section{(6) Springer}

\begin{abstract}
The conceptions of (generalized) Tykhonov well-posedness for generalized vector quasi-equilibrium problems, (generalized) Hadamard well-posedness for parametrically generalized vector quasi-equilibrium problems and (generalized) Tykhonov well-posedness for parametrical system of generalized vector quasi-equilibrium problems are introduced. The metric characterizations and/or sufficient criteria of the proposed well-posedness are presented, and the relations between (generalized) Tykhonov well-posedness for generalized vector quasi-equilibrium problems and that for constrained minimizing problems are discussed. Finally, the relations among several types of the well-posedness are exhibited in detail.
\end{abstract}

MSC: Primary 49K40; 90C31; secondary 90C33

Keywords: well-posedness; generalized vector quasi-equilibrium problem; set-valued objective mapping; relation

\section{Introduction and preliminaries}

Well-posedness is very important for both theory and numerical method of many problems such as optimization problems, optimal control, variations, mathematical programming, fixed-point problems, variational inequality, variational inclusion problems and equilibrium problems (in short, EPs), since it guarantees that for any approximating solution sequence of one of mentioned problems, there must exist a subsequence converging to some correlative solution. The classical concept of well-posedness for unconstrained optimization problem was introduced by Tykhonov [1] in Banach space in 1966. In the same year, this notion was extended to the case of constrained optimization problems by Levitin and Polyak [2]. Ever since then, various types of well-posedness for scalar or vector optimization problems with unconstraint or constraints have been widely focused on. More details on well-posedness for optimization problems, optimal control, variations and mathematical programming and for vector optimization problems can be found in the monographs [3-5] and [6], respectively. In the other directions, some kinds of well-posedness were introduced for other problems, such as fixed-point problems [7-13], variational inequality problems [11-18], vector variational inequality problems [19], variational inclusion problems [10-12, 20-22], complementary problems [23, 24], Nash EPs

( 2014 Qu and Cheng; licensee Springer. This is an Open Access article distributed under the terms of the Creative Commons Attribution License (http://creativecommons.org/licenses/by/2.0), which permits unrestricted use, distribution, and reproduction in any medium, provided the original work is properly cited. 
in the game with two players [25-27] or $n$-players [13, 28-30] and Pareto-Nash EPs in the game with finite or infinite players [31], and many significant results related to them were obtained.

As understood by Blum and Oetti [32], EPs contain many problems as special cases, for example, optimization problems, fixed-point problems, variational inequality problems, complementary problems and Nash EPs. The discussion on various aspects, such as existence of solutions, iterative algorithms and stability of solutions, etc. for these problems can be classified to the corresponding discussion for general EPs. Some results on different types of well-posedness for EPs were obtained. For instance, Long et al. [33] and Zaslavski [34] introduced the notions of generalized Levitin-Polyak well-posedness for explicit constrained EPs and generic well-posedness for EPs, respectively. Bianchi et al. [35] defined $\mathrm{T}_{\mathrm{opt}}{ }^{-}$and $\mathrm{T}_{\mathrm{vi}}$-well-posedness for EPs and proposed the conception of Hadamard wellposedness for parametrical EPs to unify two notions as above. Fang and Hu [36] and Wang and Cheng [37] defined well-posedness for parametrical systems of EPs which are the generalizations of Stampacchia/Minty type variational inequalities and quasi-variational-like inequalities. In addition, Fang et al. [38] introduced generalized well-posedness for a parametrical system of EPs. The sufficient and necessary conditions and metric characterizations of corresponding well-posedness were investigated in [33-38].

Recently, multifarious conceptions of well-posedness for vector equilibrium problems (in short, VEPs) and the related results have been recorded in many literature works. For example, the conceptions of (generalized) Levitin-Polyak well-posedness for VEPs $[39,40]$, convex symmetric vector quasi-equilibrium problems (VQEPs for brevity) [41], VQEPs without constraints [42] and VQEPs with functional constraints [43-45] were introduced respectively, and their criteria and/or metric characterizations were discussed. Besides, the notions of $M$ - and $B$-well-posedness for VEPs were presented in [46] and their sufficient conditions were given. The generalized Tykhonov well-posedness for system of VEPs was studied by Peng and Wu [47]. Also, for the well-posedness of parametric strong VQEPs, refer to [48].

Up to the present, there are few literature works to record the well-posedness for EPs involving set-valued objective mappings. The aim of this article is to explore well-posed VEPs with set-valued objective mappings. This paper is organized as follows. A generalized nonlinear scalarization function, which will be used to construct gap functions of generalized vector quasi-equilibrium problems (in short, GVQEPs), is introduced in this section. The metric characterizations and sufficient criteria of (generalized) Tykhonov wellposedness, (G)TWPness for brevity, for GVQEPs are presented by applying Kuratowski noncompactness measure, and the relations between (G)TWPness for GVQEPs and that for constrained minimizing problems are exhibited in Section 2. The sufficient conditions of (generalized) Hadamard well-posedness ((G)HWPness, for brevity) for parametrically GVQEPs are proposed in Section 3. The metric characterizations and sufficient criteria of (G)TWPness for parametrical system of GVQEPs are presented in Section 4. Finally, the relations among the types of proposed well-posedness are illuminated in detail in Section 5 .

We first recall some notions and concepts. $\mathbb{R}, \mathbb{R}_{+}$and $\mathbb{N}$ denote the sets of real numbers, non-negative real numbers and positive integers, respectively, and $\mathcal{N}(*)$ denotes the collection of all open neighborhoods of $*$, where $*$ is a point or a set in a topological space. 
Definition 1.1 Let $X$ be a topological space and $E \subset X$ be a nonempty subset. A realvalued function $g: E \rightarrow \mathbb{R}$ is said to be upper semi-continuous on $E$ if $\{x \in E: g(x)<\lambda\}$ is open for each $\lambda \in \mathbb{R}$; lower semi-continuous on $E$ if $\{x \in E: g(x)>\lambda\}$ is open for each $\lambda \in \mathbb{R}$.

Definition 1.2 ([49]) Let $X$ and $Y$ be topological spaces and $E \subset X$ be a nonempty subset. A set-valued mapping $G: E \rightarrow 2^{Y}$ is said to be upper semi-continuous at $x_{0} \in E$ if for any $N \in \mathcal{N}\left(G\left(x_{0}\right)\right)$, there exists $B \in \mathcal{N}\left(x_{0}\right)$ such that $G(x) \subset N$ for all $x \in B$; lower semicontinuous at $x_{0} \in E$ if for any $y_{0} \in G\left(x_{0}\right)$ and any $N \in \mathcal{N}\left(y_{0}\right)$, there exists $B \in \mathcal{N}\left(x_{0}\right)$ such that $G(x) \cap N \neq \emptyset$ for all $x \in B$; upper semi-continuous (resp., lower semi-continuous) on $E$ if $G$ is upper semi-continuous (resp., lower semi-continuous) at each $x \in E$; closed if its graph $\operatorname{Graph}(G)=\{(x, y) \in E \times Y: y \in G(x)\}$ is closed in $E \times Y$.

\section{Definition 1.3}

(i) Let $X$ be a topological space and $E \subset X$ be a nonempty subset. An extended real-valued function $h: E \rightarrow \mathbb{R} \cup\{+\infty\}$ is said to be level-compact on $E$ if $\{x \in E: h(x) \leq \lambda\}$ is compact for any $\lambda \in \mathbb{R}$.

(ii) Further suppose that $(X,\|\cdot\|)$ is a finite-dimensional normed linear space. $h$ is said to be level-bounded on $E$ if $E$ is bounded or

$$
\lim _{x \in E,\|x\| \rightarrow+\infty} h(x)=+\infty
$$

Lemma 1.1 Let $X$ and $Y$ be Hausdorff topological spaces and $G: X \rightarrow 2^{Y}$ be a set-valued mapping.

(i) ([49]) If $X$ is compact, and $G$ is compact-valued and upper semi-continuous on $X$, then $G(X)=\bigcup_{x \in X} G(x)$ is compact.

(ii) ([50]) If $G$ is upper semi-continuous with closed values, then $G$ is closed.

Definition 1.4 Let $(X, d)$ be a metric space and $A, B \subset X$ be nonempty subsets. The excess $\tilde{e}(A, B)$ of $A$ to $B$ and the Hausdorff distance $H(A, B)$ of $A$ and $B$ are defined as

$$
\begin{aligned}
& \tilde{e}(A, B)=\sup \{d(x, B): x \in A\}, \\
& H(A, B)=\max \{\tilde{e}(A, B), \tilde{e}(B, A)\},
\end{aligned}
$$

respectively, where $d(x, B)=\inf \{d(x, y): y \in B\}$ is the distance from $x$ to $B$.

Definition 1.5 Let $(X, d)$ be a complete metric space and $A \subset X$ be a nonempty bounded subset. The Kuratowski noncompactness measure [51] of $A$ is defined as

$$
\alpha(A)=\inf \left\{\varepsilon>0: \exists n \in \mathbb{N} \text {, s.t. } A \subset \bigcup_{i=1}^{n} A_{i}, \operatorname{diam} A_{i}<\varepsilon, \forall i=1, \ldots, n\right\},
$$

where $\operatorname{diam} A_{i}=\sup \left\{d(a, b): a, b \in A_{i}\right\}$ is the diameter of $A_{i}$. It follows from [51] that

(i) $\alpha(A)=0$ if $A$ is compact;

(ii) $\alpha(B) \leq \alpha(A)+2 \varepsilon$, where $B=\{a \in X: d(a, A)<\varepsilon\}$;

(iii) $\alpha(A)=\alpha(\operatorname{cl} A)$, where $\operatorname{cl} A$ is the closure of $A$. 
A subset $D$ of a linear space $Y$ is called a cone if $\lambda x \in D$ for all $x \in D$ and $\lambda>0$. Let $D$ be a cone in $Y$ and $A \subset Y$. D is called proper if $D \neq Y$. A is called $D$-closed [52] if $A+\operatorname{cl} D$ is closed and $D$-bounded [52] if for each neighborhood $U$ of zero in $Y$, there exists $\lambda>0$ such that $A \subset \lambda U+D$. Obviously, any compact subset in $Y$ is both $D$-closed and $D$-bounded. Let $X$ and $Y$ be nonempty sets. A set-valued mapping $G: X \rightarrow 2^{Y}$ is said to be strict if $G(x) \neq \emptyset$ for any $x \in X$.

In order to construct gap functions of GVQEPs, a generalized nonlinear scalarization function of a set-valued mapping and its properties are listed.

From now on, let $(X, d)$ be a Hausdorff complete metric space, $Y$ be a real Hausdorff topological vector space and $Z$ be a Hausdorff topological space, let $E \subset X$ and $F \subset Z$ be nonempty closed subsets, let $C: E \rightarrow 2^{Y}$ be a set-valued mapping such that $C(x)$ is a proper, closed and convex cone in $Y$ with int $C(x) \neq \emptyset$ for each $x \in E$ and let $e: E \rightarrow Y$ be a vector-valued mapping such that

$$
e(x) \in \operatorname{int} C(x) \quad \text { for all } x \in E
$$

In view of Lemma 3.1 in [53], we can define a general nonlinear scalarization function as follows.

Definition 1.6 Let $G: F \rightarrow 2^{Y}$ be a strict compact-valued mapping. A generalized nonlinear scalarization function $\zeta_{G}: E \times F \rightarrow \mathbb{R}$ of $G$ is defined by

$$
\zeta_{G}(x, u)=\min \{\lambda \in \mathbb{R}: G(u) \cap(\lambda e(x)-C(x)) \neq \emptyset\} \quad \text { for all }(x, u) \in E \times F .
$$

It is easy to find differences between the generalized nonlinear scalarization function $\zeta_{G}$ and the general nonlinear scalarization function $\xi_{G}$ given by $\mathrm{Qu}$ and Cheng [54]. But if $X=Y=Z=E=F$ and $G(u)=\{u\}$ for all $u \in F$, then both $\zeta_{G}$ and $\xi_{G}$ reduce simultaneously to the nonlinear scalarization function of a single-valued mapping introduced by Chen and Yang [55]. According to Proposition 3.1 in [53], we have the following.

Lemma 1.2 The following assertions are true for each $\lambda \in \mathbb{R}, x \in E$ and $u \in F$ :

(i) $\zeta_{G}(x, u)<\lambda \Longleftrightarrow G(u) \cap(\lambda e(x)-\operatorname{int} C(x)) \neq \emptyset$.

(ii) $\zeta_{G}(x, u) \leq \lambda \Longleftrightarrow G(u) \cap(\lambda e(x)-C(x)) \neq \emptyset$.

\section{2 (G)TWPness for GVQEPs}

In this section, the conceptions of (G)TWPness for GVQEPs are introduced, their metric characterizations are depicted by using Kuratowski noncompactness measure, and some sufficient criteria are presented. Besides, the relations between (G)TWPness for GVQEPs and that for constrained minimizing problems are exhibited. The GVQEP is defined as

(GVQEP) to find $\bar{x} \in P(\bar{x})$ such that $f(\bar{x}, z) \cap(-\operatorname{int} C(\bar{x}))=\emptyset$ for all $z \in Q(\bar{x})$,

where $f: E \times F \rightarrow 2^{Y}, P: E \rightarrow 2^{E}$ and $Q: E \rightarrow 2^{F}$ are strict set-valued mappings. $\Omega$ denotes the solution set of (GVQEP).

If $E=F=X=Z, f$ is single-valued and $P(x)=Q(x)=E$ for all $x \in E$, then (GVQEP) reduces to $V E P$ described as:

(VEP) to find $\bar{x} \in E$ such that $f(\bar{x}, z) \notin-\operatorname{int} C(\bar{x})$ for all $z \in E$. 
For each $\varepsilon \geq 0$, the following assumptions are introduced:

$$
\begin{aligned}
& d(x, P(x)) \leq \varepsilon, \\
& f(x, z) \cap(-\varepsilon e(x)-\operatorname{int} C(x))=\emptyset \quad \text { for all } z \in Q(x), \\
& f(x, \tilde{z}) \cap(\varepsilon e(x)-C(x)) \neq \emptyset \quad \text { for some } \tilde{z} \in Q(x) .
\end{aligned}
$$

Definition 2.1 A type I $\varepsilon$-approximating solution set (resp., type II E-approximating solution set) of (GVQEP) is defined by

$$
\Omega_{1}(\varepsilon)=\{x \in E: x \text { satisfies }(2.1) \text { and }(2.2)\}
$$

(resp., $\Omega_{2}(\varepsilon)=\{x \in E: x$ satisfies $\left.(2.1)-(2.3)\}\right)$.

A sequence $\left\{x_{n}\right\}$ is called a type I approximating solution sequence, ASS1 for brevity (resp., type II approximating solution sequence, ASS2 for brevity) of (GVQEP) if there exists $\left\{\varepsilon_{n}\right\} \subset \mathbb{R}_{+}$with $\varepsilon_{n} \rightarrow 0$ such that $x_{n} \in \Omega_{1}\left(\varepsilon_{n}\right)$ (resp., $x_{n} \in \Omega_{2}\left(\varepsilon_{n}\right)$ ).

Definition 2.2 (GVQEP) is said to be generalized type I Tykhonov well-posed, GTWP1 for brevity (resp., generalized type II Tykhonov well-posed, GTWP2 for brevity) if $\Omega \neq \emptyset$ and for any ASS1 (resp., ASS2) $\left\{x_{n}\right\}$ of (GVQEP), there exists a subsequence $\left\{x_{n_{i}}\right\}$ such that $x_{n_{i}} \rightarrow \bar{x} \in \Omega$; to be type I Tykhonov well-posed, TWP1 for brevity (resp., type II Tykhonov well-posed, TWP2 for brevity) if it is GTWP1 (resp., GTWP2) and $\Omega$ is a singleton.

When (GVQEP) reduces to (VEP), generalized type I Tykhonov well-posedness (GTWPness1 for brevity) and generalized type II Tykhonov well-posedness (GTWPness2 for brevity) for (GVQEP) become type I Levitin-Polyak well-posedness and type II LevitinPolyak well-posedness for (VEP), respectively, which were discussed by Li and Li [39] in the case that $X$ and $Y$ are locally convex topological vector spaces, where $X$ is equipped with a metric $d$ compatible with its topology, $F$ is a nonempty closed convex subset, and $f$ is a continuous mapping.

Remark 2.1 (i) An ASS2 of (GVQEP) must be its ASS1. So GTWPness1 (resp., TWPness1) for (GVQEP) implies its GTWPness2 (resp., TWPness2), where TWPness1 and TWPness2 are the abbreviations of type I Tykhonov well-posedness and type II Tykhonov well-posedness, respectively.

(ii) Clearly, $\Omega_{1}(0)=\Omega$ if $P$ is closed-valued. In addition, $\Omega \subset \Omega_{1}(\varepsilon)$ for all $\varepsilon \geq 0$. In fact, for any $\bar{x} \in \Omega$, we have $\bar{x} \in P(\bar{x})$ and

$$
f(\bar{x}, z) \cap(-\operatorname{int} C(\bar{x}))=\emptyset \quad \text { for all } z \in Q(\bar{x}) .
$$

Then $d(\bar{x}, P(\bar{x})) \leq \varepsilon$ for any $\varepsilon \geq 0$. It follows from (2.4) and (1.1) that (2.2) holds.

(iii) (GVQEP) is GTWP1 if and only if $\Omega$ is nonempty compact and $d\left(x_{n}, \Omega\right) \rightarrow 0$ for its any ASS1 $\left\{x_{n}\right\}$. Assume that $\Omega$ is compact, (GVQEP) is GTWP2 if and only if $\Omega \neq \emptyset$ and $d\left(x_{n}, \Omega\right) \rightarrow 0$ for its any ASS2 $\left\{x_{n}\right\}$. In addition, (GVQEP) is TWP1 (resp., TWP2) if and only if $\Omega=\{\bar{x}\}$ and $d\left(x_{n}, \bar{x}\right) \rightarrow 0$ for any ASS1 (resp., ASS2) $\left\{x_{n}\right\}$ of (GVQEP).

The following example shows that neither the GTWPness1 for (GVQEP) nor the compactness of $\Omega$ can be deduced from the GTWPness2 for (GVQEP). 
Example 2.1 Let $E=X=Y=Z=\mathbb{R}, F=\mathbb{R}_{+}, P(x)=\{x\}, Q(x)=C(x)=\mathbb{R}_{+}, e(x)=1$ for all $x \in \mathbb{R}$ and

$$
f(x, z)= \begin{cases}{[-x+z,-x+z+1]} & \text { if } x \in(-\infty, 0], z \in F \\ {[x+z-2 k+1, x+z-2 k+2]} & \text { if } x \in(2 k, 2 k+2], k=0,1,2, \ldots, z \in F .\end{cases}
$$

(GVQEP) is to find $\bar{x} \in \mathbb{R}$ such that

$$
f(\bar{x}, z) \cap(-\infty, 0)=\emptyset \quad \text { for all } z \in \mathbb{R}_{+} .
$$

Obviously, $\Omega=\mathbb{R}$ is noncompact and so (GVQEP) is not GTWP1 by Remark 2.1(iii). However, (GVQEP) is GTWP2. In fact, for any ASS2 $\left\{x_{n}\right\}$ of (GVQEP), let $\left\{\varepsilon_{n}\right\} \subset \mathbb{R}_{+}$with $\varepsilon_{n} \rightarrow 0$ such that $x_{n} \in \Omega_{2}\left(\varepsilon_{n}\right)$. For $x_{n}$ and $\varepsilon_{n},(2.1)$ and (2.2) hold trivially.

It is impossible that $x_{n}>0$ for sufficiently large $n \in \mathbb{N}$. Otherwise, $x_{n} \in(2 k, 2 k+2]$ for some $k \in\{0,1,2, \ldots\}$. By (2.3), there exists $\tilde{z}_{n} \in \mathbb{R}_{+}$such that $x_{n}+\tilde{z}_{n}-2 k+1-\varepsilon_{n} \leq 0$. We have

$$
2 k<x_{n} \leq-\tilde{z}_{n}+2 k-1+\varepsilon_{n} \leq 2 k-1+\varepsilon_{n} .
$$

This is absurd for sufficiently large $n$. Therefore, without loss of generality, $\left\{x_{n}\right\} \subset(-\infty, 0]$. It follows from (2.3) that there exists $\tilde{z}_{n} \in \mathbb{R}_{+}$such that $-x_{n}+\tilde{z}_{n}-\varepsilon_{n} \leq 0$. Then

$$
-\varepsilon_{n} \leq \tilde{z}_{n}-\varepsilon_{n} \leq x_{n} \leq 0 .
$$

The fact $x_{n} \rightarrow 0 \in \Omega$ proceeds from (2.5) and $\varepsilon_{n} \rightarrow 0$.

\subsection{Metric characterization of (G)TWPness for (GVQEP)}

The metric characterizations of (G)TWPness for GVQEPs are depicted by using Kuratowski noncompactness measure and the corresponding results are obtained as follows.

\section{Lemma 2.1 Suppose that}

(a1) $f$ is lower semi-continuous on $E \times F$;

(a2) $P$ is compact-valued and upper semi-continuous on $E$;

(a3) $Q$ is lower semi-continuous on $E$;

(a4) $W$ is upper semi-continuous on $E$, where $W: E \rightarrow 2^{Y}$ is defined as

$$
W(x)=Y \backslash-\operatorname{int} C(x) \text { for all } x \in E ;
$$

(a5) $e$ is continuous on $E$.

\section{Then}

(i) $\Omega_{1}(\varepsilon)$ is closed for each $\varepsilon>0$;

(ii) $\Omega=\bigcap_{\varepsilon>0} \Omega_{1}(\varepsilon)$.

Proof (i) For each fixed $\varepsilon>0$, assume that $\left\{x_{n}\right\} \subset \Omega_{1}(\varepsilon)$ with $x_{n} \rightarrow \bar{x}$. Then

$$
\begin{aligned}
& d\left(x_{n}, P\left(x_{n}\right)\right) \leq \varepsilon, \\
& f\left(x_{n}, z_{n}\right) \cap\left(-\varepsilon e\left(x_{n}\right)-\operatorname{int} C\left(x_{n}\right)\right)=\emptyset \quad \text { for all } z_{n} \in Q\left(x_{n}\right) .
\end{aligned}
$$


As a result of (a2) and Lemma 1.1(ii), $P$ is closed. Letting $n \rightarrow+\infty$ in (2.6), we have

$$
d(\bar{x}, P(\bar{x})) \leq \varepsilon .
$$

As a matter of fact, if we take $A=\left\{\bar{x}, x_{1}, x_{2}, x_{3}, \ldots\right\}$, then $A$ is compact and so is $P(A)$ by Lemma 1.1(i). Since $P\left(x_{n}\right)$ is compact, there exists $y_{n} \in P\left(x_{n}\right)$ such that $d\left(x_{n}, y_{n}\right)=$ $d\left(x_{n}, P\left(x_{n}\right)\right)$ and some subsequence of $\left\{y_{n}\right\}$, still denoted by $\left\{y_{n}\right\}$, converging to some point $\bar{y} \in P(\bar{x})$ by $\left\{y_{n}\right\} \subset P(A)$, the compactness of $P(A)$ and the closeness of $P$. Thus,

$$
d(\bar{x}, P(\bar{x})) \leq d(\bar{x}, \bar{y})=\lim _{n \rightarrow+\infty} d\left(x_{n}, y_{n}\right)=\lim _{n \rightarrow+\infty} d\left(x_{n}, P\left(x_{n}\right)\right) \leq \varepsilon .
$$

For any $z \in Q(\bar{x})$, there exists $\tilde{z}_{n} \in Q\left(x_{n}\right)$ such that $\tilde{z}_{n} \rightarrow z$ by virtue of (a3). Likewise, for any $y \in f(\bar{x}, z)$, there exists $\tilde{y}_{n} \in f\left(x_{n}, \tilde{z}_{n}\right)$ such that $\tilde{y}_{n} \rightarrow y$ by (a1). This, together with (2.7), implies that $\tilde{y}_{n} \notin-\varepsilon e\left(x_{n}\right)-\operatorname{int} C\left(x_{n}\right)$, in other words,

$$
\tilde{y}_{n} \in-\varepsilon e\left(x_{n}\right)+W\left(x_{n}\right) .
$$

It is easy to see that $W$ is closed by Lemma 1.1. It follows that $y \in-\varepsilon e(\bar{x})+W(\bar{x})$ from (2.9), the continuity of $e$ and the closeness of $W$, and so

$$
f(\bar{x}, z) \cap(-\varepsilon e(\bar{x})-\operatorname{int} C(\bar{x}))=\emptyset \quad \text { for all } z \in Q(\bar{x}) .
$$

Thus $\bar{x} \in \Omega_{1}(\varepsilon)$ and $\Omega_{1}(\varepsilon)$ is closed.

(ii) $\Omega \subset \bigcap_{\varepsilon>0} \Omega_{1}(\varepsilon)$ stems easily from Remark 2.1(ii). For any $\bar{x} \in \bigcap_{\varepsilon>0} \Omega_{1}(\varepsilon)$, (2.8) and (2.10) hold for any $\varepsilon>0$. Then $\bar{x} \in P(\bar{x})$ by (a2), and $y+\varepsilon e(\bar{x}) \in W(\bar{x})$ for any $y \in f(\bar{x}, z)$ and $z \in Q(\bar{x})$ by (2.10). Letting $\varepsilon \rightarrow 0$, we have $y \in W(\bar{x})$, and so $f(\bar{x}, z) \cap(-\operatorname{int} C(\bar{x}))=\emptyset$. Consequently, $\bar{x} \in \Omega$ and $\bigcap_{\varepsilon>0} \Omega_{1}(\varepsilon) \subset \Omega$.

Lemma 2.2 Suppose that (a1)-(a5) and

(a6) $0 \in f(x, Q(x))$ for all $x \in E$

hold. Then

(i) $\Omega_{2}(\varepsilon)$ is closed for each $\varepsilon>0$;

(ii) $\Omega=\bigcap_{\varepsilon>0} \Omega_{2}(\varepsilon)$.

Proof (i) For each $\varepsilon>0$, let $\left\{x_{n}\right\} \subset \Omega_{2}(\varepsilon)$ with $x_{n} \rightarrow \bar{x}$. It is enough to testify that $\bar{x}$ satisfies (2.3) by Lemma 2.1(i). As a matter of fact, $0 \in f(\bar{x}, \tilde{z})$ for some $\tilde{z} \in Q(\bar{x})$ according to (a6). As a result, $f(\bar{x}, \tilde{z}) \cap(\varepsilon e(\bar{x})-C(\bar{x})) \neq \emptyset$ owing to (1.1).

(ii) $\Omega=\bigcap_{\varepsilon>0} \Omega_{1}(\varepsilon) \supset \bigcap_{\varepsilon>0} \Omega_{2}(\varepsilon)$ by Lemma 2.1(ii). We only need to show that $\bar{x}$ satisfies

(2.3) for any $\bar{x} \in \Omega$ and $\varepsilon>0$, while this can be deduced easily from the proof of (i).

Theorem 2.1 Suppose that $E$ is bounded.

(i) If (GVQEP) is GTWP1, then

$$
\Omega_{1}(\varepsilon) \neq \emptyset \quad \text { for all } \varepsilon>0 \text { and } \lim _{\varepsilon \rightarrow 0} \alpha\left(\Omega_{1}(\varepsilon)\right)=0 \text {. }
$$

(ii) If (a1)-(a5) hold, then (2.11) implies that (GVQEP) is GTWP1. 
Proof (i) Since (GVQEP) is GTWP1, $\Omega$ is nonempty compact and so $\Omega_{1}(\varepsilon) \neq \emptyset$ for all $\varepsilon>0$. Also $\alpha(\Omega)=0$ by the compactness of $\Omega$ and $\Omega \subset \Omega_{1}(\varepsilon)$ by Remark 2.1(ii). This deduces that

$$
\alpha\left(\Omega_{1}(\varepsilon)\right) \leq 2 \tilde{e}\left(\Omega_{1}(\varepsilon), \Omega\right)+\alpha(\Omega)=2 \tilde{e}\left(\Omega_{1}(\varepsilon), \Omega\right) .
$$

It is enough to testify that $\tilde{e}\left(\Omega_{1}(\varepsilon), \Omega\right) \rightarrow 0$ as $\varepsilon \rightarrow 0$. Otherwise, there exist $r>0, \varepsilon_{n} \downarrow 0$ and $x_{n} \in \Omega_{1}\left(\varepsilon_{n}\right)$ such that $d\left(x_{n}, \Omega\right) \geq r$ for all $n \in \mathbb{N}$. Clearly, $\left\{x_{n}\right\}$ is an ASS1 of (GVQEP). Thus $d\left(x_{n}, \Omega\right) \rightarrow 0$ by Remark 2.1(iii), which contradicts $d\left(x_{n}, \Omega\right) \geq r$ for all $n \in \mathbb{N}$.

(ii) For any ASS1 $\left\{x_{n}\right\}$ of (GVQEP), let $\left\{\varepsilon_{n}\right\} \subset \mathbb{R}_{+}$with $\varepsilon_{n} \rightarrow 0$ such that $x_{n} \in \Omega_{1}\left(\varepsilon_{n}\right)$. In view of Lemma 2.1 and the boundedness of $E, \lim _{\varepsilon \rightarrow 0} \Omega_{1}(\varepsilon)=\Omega$ and $\Omega_{1}(\varepsilon)$ is a nonempty bounded closed set. For any $0<\varepsilon_{1}<\varepsilon_{2}$ and $x \in E$,

$$
-\varepsilon_{2} e(x)-\operatorname{int} C(x)=-\varepsilon_{1} e(x)-\left(\varepsilon_{2}-\varepsilon_{1}\right) e(x)-\operatorname{int} C(x) \subset-\varepsilon_{1} e(x)-\operatorname{int} C(x)
$$

by (1.1). Therefore, $\Omega_{1}\left(\varepsilon_{1}\right) \subset \Omega_{1}\left(\varepsilon_{2}\right)$, and so $\Omega_{1}(\cdot)$ is increasing with $\varepsilon>0$. This, together with $\lim _{\varepsilon \rightarrow 0} \alpha\left(\Omega_{1}(\varepsilon)\right)=0$, implies that $\Omega$ is nonempty compact and

$$
H\left(\Omega_{1}(\varepsilon), \Omega\right) \rightarrow 0 \quad \text { as } \varepsilon \rightarrow 0
$$

by Kuratowski theorem [51]. Resultingly, $d\left(x_{n}, \Omega\right) \rightarrow 0$ and (GVQEP) is GTWP1 by Remark 2.1(iii).

Similarly, the following result can be proved by using Lemma 2.2 .

Theorem 2.2 Assume that $E$ is bounded and $\Omega$ is compact.

(i) If (GVQEP) is GTWP2, then

$$
\Omega_{2}(\varepsilon) \neq \emptyset \quad \text { for all } \varepsilon>0 \text { and } \lim _{\varepsilon \rightarrow 0} \alpha\left(\Omega_{2}(\varepsilon)\right)=0 \text {. }
$$

(ii) If (a1)-(a6) are satisfied, then (2.12) implies that (GVQEP) is GTWP2.

When $\Omega$ is a singleton, the following corollary that shows the metric information of TWPness1 and TWPness2 for (GVQEP) follows from Theorems 2.1 and 2.2.

Corollary 2.1 Suppose that $E$ is bounded and $\Omega$ is a singleton.

(i) If (GVQEP) is TWP1 (resp., TWP2), then (2.11) (resp., (2.12)) holds.

(ii) If (a1)-(a5) (resp., (a1)-(a6)) hold, then (2.11) (resp., (2.12)) implies that (GVQEP) is $T W P 1$ (resp., TWP2).

\subsection{Relations between (G)TWPness for (GVQEP) and that for constrained minimizing problem}

First, we introduce the constrained minimizing problem described as follows:

(CMP) $\min \phi(x)$ subject to $x \in P(x)$,

where $\phi: E \rightarrow \mathbb{R} \cup\{+\infty\}$ is a proper function and $P: E \rightarrow 2^{E}$ is a strict set-valued mapping. The optimal set and optimal value of (CMP) are denoted by $\operatorname{argmin} \phi$ and $\tilde{v}$, respectively. In 
this subsection, the equivalent relations between (G)TWPness for (GVQEP) and that for (CMP) are discussed, where a gap function of (GVQEP) is taken as the objective function $\phi$ of (CMP).

Definition 2.3 A sequence $\left\{x_{n}\right\}$ is called a type I minimizing sequence, MS1 for brevity (resp., type II minimizing sequence, MS2 for brevity) of (CMP) if the following (2.13) and (2.14) (resp., (2.13) and (2.15)) hold.

$$
\begin{aligned}
& \lim _{n \rightarrow+\infty} d\left(x_{n}, P\left(x_{n}\right)\right)=0, \\
& \limsup _{n \rightarrow+\infty} \phi\left(x_{n}\right) \leq \tilde{v}, \\
& \limsup _{n \rightarrow+\infty} \phi\left(x_{n}\right)=\tilde{v} .
\end{aligned}
$$

Definition 2.4 (CMP) is said to be GTWP1 (resp., GTWP2) if $\operatorname{argmin} \phi \neq \emptyset$ and for any MS1 (resp., MS2) $\left\{x_{n}\right\}$ of (CMP), there exists a subsequence $\left\{x_{n_{i}}\right\}$ such that $x_{n_{i}} \rightarrow \bar{x} \in$ $\operatorname{argmin} \phi$; to be TWP1 (resp., TWP2) if it is GTWP1 (resp., GTWP2) and $\operatorname{argmin} \phi$ is a singleton.

Definition 2.5 $g: E \rightarrow \mathbb{R} \cup\{+\infty\}$ is called a gap function of (GVQEP) if

(i) $g(x) \geq 0$ for all $x \in E$;

(ii) $x \in\{u \in E: g(u)=0$ and $u \in P(u)\}$ if and only if $x \in \Omega$.

Further suppose that $f$ is compact-valued in this subsection.

Lemma 2.3 If (a6) holds, then $\phi$ is a gap function of (GVQEP), where $\phi: E \rightarrow \mathbb{R} \cup\{+\infty\}$ is defined by

$$
\phi(x)=\sup _{z \in Q(x)}-\zeta_{f}(x,(x, z)) \quad \text { for all } x \in E,
$$

and

$$
\zeta_{f}(x,(u, z))=\min \{\lambda \in \mathbb{R}: f(u, z) \cap(\lambda e(x)-C(x)) \neq \emptyset\} \quad \text { for all } x, u \in E \text { and } z \in F .
$$

Proof Clearly, $\phi(x)>-\infty$ for all $x \in E$. Otherwise, $\phi(\bar{x})=-\infty$ for some $\bar{x} \in E$. Then $\zeta_{f}(\bar{x},(\bar{x}, \bar{z})) \geq+\infty$ for all $\bar{z} \in Q(\bar{x})$, which contradicts the fact that $\zeta_{f}$ is real-valued.

It follows from (a6) and Lemma 1.2(ii) that for each $x \in E, \zeta_{f}(x,(x, \tilde{z})) \leq 0$ for some $\tilde{z} \in$ $Q(x)$. This deduces that

$$
\phi(x)=\sup _{z \in Q(x)}-\zeta_{f}(x,(x, z)) \geq 0 .
$$

Finally, since

$$
\begin{aligned}
& x \in P(x), \quad \phi(x)=\sup _{z \in Q(x)}-\zeta_{f}(x,(x, z))=0 \\
& \Longleftrightarrow x \in P(x), \zeta_{f}(x,(x, z)) \geq 0 \text { for all } z \in Q(x)(\text { By }(2.17)) ;
\end{aligned}
$$


$\Longleftrightarrow x \in P(x), f(x, z) \cap(-$ int $C(x))=\emptyset$ for all $z \in Q(x)$ (By Lemma 1.2(i));

$\Longleftrightarrow x \in \Omega$,

$\phi$ is a gap function of (GVQEP).

In general, $\phi$ is required to be lower semi-continuous. It is natural to expect the lower semi-continuity of the constructed gap function. Now assume that $\phi$ appearing in the rest of this section is defined as (2.16).

Proposition 2.1 If (a1)-(a5) hold, then $\phi$ is lower semi-continuous on E. If, further, (a6) holds and $\Omega \neq \emptyset$, then $\operatorname{dom} \phi \neq \emptyset$.

Proof In order to verify that $\phi$ is lower semi-continuous on $E$, it is enough to show that $L(\varepsilon)=\{x \in E: \phi(x) \leq \varepsilon\}$ is closed for each $\varepsilon \in \mathbb{R}$. In fact, let $\left\{x_{n}\right\} \subset L(\varepsilon)$ with $x_{n} \rightarrow \bar{x}$. Then

$$
\zeta_{f}\left(x_{n},\left(x_{n}, z_{n}\right)\right) \geq-\varepsilon \text { for all } z_{n} \in Q\left(x_{n}\right)
$$

and (2.7) holds by Lemma 1.2(i). It is easy to see that (2.10) holds by (2.7) and a similar argument given in the proof of Lemma 2.1. Applying Lemma 1.2(i) again, we have

$$
\zeta_{f}(\bar{x},(\bar{x}, z)) \geq-\varepsilon \quad \text { for all } z \in Q(\bar{x})
$$

that is, $\bar{x} \in L(\varepsilon)$. If, further, (a6) holds and $\Omega \neq \emptyset$, then $\operatorname{dom} \phi \neq \emptyset$ by Lemma 2.3 and Definition 2.5 .

Theorem 2.3 Assume that (a6) holds. Then (GVQEP) is GTWP1 (resp., GTWP2) if and only if so is (CMP) with the objective function $\phi$.

Proof $\phi$ is a gap function of (GVQEP) owing to Lemma 2.3. Thus $\bar{x} \in \Omega$ if and only if $\bar{x} \in$ $\operatorname{argmin} \phi$. Here, $\tilde{v}=0$. Two equivalent relations are listed as follows:

$1^{\circ}\left\{x_{n}\right\}$ is an ASS1 of (GVQEP).

$\Longleftrightarrow$ There exists $\left\{\varepsilon_{n}\right\} \subset \mathbb{R}_{+}$with $\varepsilon_{n} \rightarrow 0$ such that $x_{n} \in \Omega_{1}\left(\varepsilon_{n}\right)$;

$\Longleftrightarrow$ There exists $\left\{\varepsilon_{n}\right\} \subset \mathbb{R}_{+}$with $\varepsilon_{n} \rightarrow 0$ such that $d\left(x_{n}, P\left(x_{n}\right)\right) \leq \varepsilon_{n}$ and

$\zeta_{f}\left(x_{n},\left(x_{n}, z_{n}\right)\right) \geq-\varepsilon_{n}$ for all $z_{n} \in Q\left(x_{n}\right)$. (By Lemma 1.2(i));

$\Longleftrightarrow$ There exists $\left\{\varepsilon_{n}\right\} \subset \mathbb{R}_{+}$with $\varepsilon_{n} \rightarrow 0$ such that $d\left(x_{n}, P\left(x_{n}\right)\right) \leq \varepsilon_{n}$ and

$$
\begin{aligned}
& \phi\left(x_{n}\right)=\sup _{z_{n} \in Q\left(x_{n}\right)}-\zeta_{f}\left(x_{n},\left(x_{n}, z_{n}\right)\right) \leq \varepsilon_{n} ; \\
& \Longleftrightarrow \lim _{n \rightarrow+\infty} d\left(x_{n}, P\left(x_{n}\right)\right)=0 \text { and } \lim \sup _{n \rightarrow+\infty} \phi\left(x_{n}\right) \leq \tilde{v}=0 ; \\
& \Longleftrightarrow\left\{x_{n}\right\} \text { is an MS1 of }(\mathrm{CMP}) . \\
& 2^{\circ}\left\{x_{n}\right\} \text { is an ASS } 2 \text { of }(\mathrm{GVQEP}) . \\
& \Longleftrightarrow \text { There exists }\left\{\varepsilon_{n}\right\} \subset \mathbb{R}_{+} \text {with } \varepsilon_{n} \rightarrow 0 \text { such that } x_{n} \in \Omega_{2}\left(\varepsilon_{n}\right) ; \\
& \Longleftrightarrow \text { There exists }\left\{\varepsilon_{n}\right\} \subset \mathbb{R}_{+} \text {with } \varepsilon_{n} \rightarrow 0 \text { such that } x_{n} \in \Omega_{1}\left(\varepsilon_{n}\right) \text { and } \\
& f\left(x_{n}, \tilde{z}_{n}\right) \cap\left(\varepsilon_{n} e\left(x_{n}\right)-C\left(x_{n}\right)\right) \neq \emptyset \text { for some } \tilde{z}_{n} \in Q\left(x_{n}\right) ; \\
& \Longleftrightarrow(\mathrm{A}): \text { There exists }\left\{\varepsilon_{n}\right\} \subset \mathbb{R}_{+} \text {with } \varepsilon_{n} \rightarrow 0 \text { such that } x_{n} \in \Omega_{1}\left(\varepsilon_{n}\right) \text { and } \\
&\left.\zeta_{f}\left(x_{n},\left(x_{n}, \tilde{z}_{n}\right)\right) \leq \varepsilon_{n} \text { for some } \tilde{z}_{n} \in Q\left(x_{n}\right) \text {. (By Lemma } 1.2(\mathrm{ii})\right) ;
\end{aligned}
$$


$\Longleftrightarrow(\mathrm{B})$ : There exists $\left\{\beta_{n}\right\} \subset \mathbb{R}_{+}$with $\beta_{n} \rightarrow 0$ such that $x_{n} \in \Omega_{1}\left(\beta_{n}\right)$ and

$$
\begin{aligned}
& \phi\left(x_{n}\right)=\sup _{z_{n} \in Q\left(x_{n}\right)}-\zeta_{f}\left(x_{n},\left(x_{n}, z_{n}\right)\right) \geq-\beta_{n} ; \\
\Longleftrightarrow & \left.\lim _{n \rightarrow+\infty} d\left(x_{n}, P\left(x_{n}\right)\right)=0 \text { and } \lim \sup _{n \rightarrow+\infty} \phi\left(x_{n}\right)=0 \text {. (By } 1^{\circ}\right) ; \\
\Longleftrightarrow & \left\{x_{n}\right\} \text { is an MS2 of }(\mathrm{CMP}) .
\end{aligned}
$$

Now we shall prove the equivalence of (A) and (B). In fact, (A) implies (B) by taking $\beta_{n}=\varepsilon_{n}$. On the other hand, if (B) holds, then for fixed $n \in \mathbb{N}$ and for any $\gamma_{n}>0$, there exists $z_{n} \in Q\left(x_{n}\right)$ such that

$$
-\beta_{n}-\gamma_{n} \leq \phi\left(x_{n}\right)-\gamma_{n}<-\zeta_{f}\left(x_{n},\left(x_{n}, z_{n}\right)\right) .
$$

We can choose $\gamma_{n} \rightarrow 0$ and $\tilde{z}_{n} \in Q\left(x_{n}\right)$ is the corresponding point such that the above inequality holds. Therefore, (A) holds by taking $\varepsilon_{n}=\beta_{n}+\gamma_{n}$.

It follows from $1^{\circ}$ (resp., $2^{\circ}$ ) that (GVQEP) is GTWP1 (resp., GTWP2) if and only if so is (CMP).

Corollary 2.2 Assume that (a6) holds. Then (GVQEP) is TWP1 (resp., TWP2) if and only if so is (CMP) with the objective function $\phi$.

When the assumptions in Theorem 2.3 are satisfied, we see that if (GVQEP) is GTWP1 (resp., GTWP2), then for any MS1 (resp., MS2) $\left\{x_{n}\right\}$ of (CMP) and for some $\bar{x} \in \operatorname{argmin} \phi=\Omega, \limsup _{n \rightarrow+\infty} \phi\left(x_{n}\right) \leq \phi(\bar{x})=\tilde{v}$ (resp., $\left.\limsup _{n \rightarrow+\infty} \phi\left(x_{n}\right)=\phi(\bar{x})=\tilde{v}\right)$ implies that $d\left(x_{n}, \bar{x}\right) \rightarrow 0$, that is, $d\left(x_{n}, \Omega\right) \rightarrow 0$. It is reasonable to try estimating a bound below of $|\phi(x)-\tilde{v}|$ by using $d(x, \Omega)$. For the sake of this intention, a forcing function with parameter is introduced.

A real-valued bifunction $c: S \times T \rightarrow \mathbb{R}_{+}$is called a forcing function with parameter (where $S$ is a parameter set) if

$$
\begin{array}{ll}
0 \in S, \quad T \subset \mathbb{R}_{+}, \quad c(0,0)=0, \\
s_{n} \rightarrow 0, \quad t_{n} \in T, \quad c\left(s_{n}, t_{n}\right) \rightarrow 0 \quad \Longrightarrow \quad t_{n} \rightarrow 0 .
\end{array}
$$

Theorem 2.4 Suppose that (a6) holds and $\phi$ is the objective mapping of (CMP). Then the following assertions are equivalent:

(i) (GVQEP) is GTWP1;

(ii) $\Omega$ is nonempty compact and there exists a forcing function with parameter $c: S \times T \rightarrow \mathbb{R}_{+}$(where $S$ is the parameter set) such that

$$
\phi(x) \geq c(d(x, P(x)), d(x, \Omega)) \text { for all } x \in E,
$$

where

$$
S=\{d(x, P(x)): x \in E\} \quad \text { and } \quad T=\{d(x, \Omega): x \in E\} .
$$

Proof By virtue of Lemma 2.3, $\phi$ is a gap function of (GVQEP). 
Let (i) hold. $\Omega$ is nonempty compact by Remark 2.1(iii). Define $c: S \times T \rightarrow \mathbb{R}_{+}$as

$$
c(s, t)=\inf \{\phi(x): d(x, P(x))=s, d(x, \Omega)=t\},
$$

where $S$ and $T$ are defined by (2.21). If $s=t=0$, then $x \in \Omega$ by the compactness of $\Omega$ and so $\phi(x)=0$ according to Definition 2.5(ii). So $c(0,0)=0$, that is, $c$ satisfies (2.18). Let $s_{n} \rightarrow 0$ and $t_{n} \in T$ with $c\left(s_{n}, t_{n}\right) \rightarrow 0$. Since

$$
c\left(s_{n}, t_{n}\right)=\inf \left\{\phi(x): d(x, P(x))=s_{n}, d(x, \Omega)=t_{n}\right\},
$$

there exists $\left\{x_{n}\right\} \subset E$ such that $s_{n}=d\left(x_{n}, P\left(x_{n}\right)\right) \rightarrow 0, t_{n}=d\left(x_{n}, \Omega\right)$ and $\phi\left(x_{n}\right) \rightarrow 0$ by the definition of infimum. Since $\tilde{v}=0,\left\{x_{n}\right\}$ is an MS1 of (CMP) and also an ASS1 of (GVQEP) in view of the proof of Theorem 2.3. Then (2.19) follows from the GTWPness1 for (GVQEP) and Remark 2.1(iii). Therefore, the assertion (ii) is true.

Suppose that (ii) holds. For any ASS1 $\left\{x_{n}\right\}$ of (GVQEP), (2.20) deduces

$$
\phi\left(x_{n}\right) \geq c\left(d\left(x_{n}, P\left(x_{n}\right)\right), d\left(x_{n}, \Omega\right)\right) \quad \text { for all } n \in \mathbb{N} \text {. }
$$

Setting $s_{n}=d\left(x_{n}, P\left(x_{n}\right)\right)$ and $t_{n}=d\left(x_{n}, \Omega\right)$, we have $s_{n} \rightarrow 0$. By the same argument given in the proof of Theorem 2.3, $\left\{x_{n}\right\}$ is an MS1 of (CMP). Therefore, $\lim _{\sup _{n \rightarrow+\infty}} \phi\left(x_{n}\right) \leq 0$. On the other hand, $\liminf _{n \rightarrow+\infty} \phi\left(x_{n}\right) \geq 0$ since $\phi\left(x_{n}\right) \geq 0$ for all $n \in \mathbb{N}$. Thus $\phi\left(x_{n}\right) \rightarrow 0$ and $c\left(s_{n}, t_{n}\right) \rightarrow 0$, and so $t_{n}=d\left(x_{n}, \Omega\right) \rightarrow 0$ by (2.19). This, together with the compactness of $\Omega$ and Remark 2.1(iii), implies that (GVQEP) is GTWP1.

Similarly, we can prove the following result by using Remark 2.1(iii).

Theorem 2.5 If (a6) holds and $\Omega$ is compact, then the following assertions are equivalent:

(i) (GVQEP) is GTWP2;

(ii) $\Omega \neq \emptyset$ and there exists a forcing function with parameter $c: S \times T \rightarrow \mathbb{R}_{+}$(where $S$ is the parameter set) such that (2.20) holds, where $S$ and $T$ are defined by (2.21).

Corollary 2.3 If (a6) holds, then the following assertions are equivalent:

(i) (GVQEP) is TWP1 (resp., TWP2);

(ii) $\Omega$ is a singleton and there exists a forcing function with parameter c: $S \times T \rightarrow \mathbb{R}_{+}$ (where $S$ is the parameter set) such that (2.20) holds, where $S$ and $T$ are defined by (2.21).

\subsection{Sufficient criteria of (G)TWP for (GVQEP)}

In this subsection, we shall list some sufficient criteria of (G)TWPness for (GVQEP).

Theorem 2.6 Let (a1)-(a5) hold and $\Omega \neq \emptyset$. If

(b1) $\Omega_{1}\left(\varepsilon_{0}\right)$ is compact for some $\varepsilon_{0}>0$

holds, then (GVQEP) is GTWP1 and also GTWP2.

Proof For any ASS1 $\left\{x_{n}\right\}$ of (GVQEP), let $\left\{\varepsilon_{n}\right\} \subset \mathbb{R}_{+}$with $\varepsilon_{n} \rightarrow 0$ such that $x_{n} \in \Omega_{1}\left(\varepsilon_{n}\right)$.

Since $\varepsilon_{n} \rightarrow 0, x_{n} \in \Omega_{1}\left(\varepsilon_{0}\right)$ for sufficiently large $n \in \mathbb{N}$. So $\left\{x_{n}\right\}$ has a subsequence, still denoted by $\left\{x_{n}\right\}$, such that $x_{n} \rightarrow \bar{x} \in E$. It follows from (a2) that $\bar{x} \in P(\bar{x})$. For any 
$z \in Q(\bar{x})$, there exists $\tilde{z}_{n} \in Q\left(x_{n}\right)$ such that $\tilde{z}_{n} \rightarrow z$ by (a3). For each $y_{n} \in f\left(x_{n}, \tilde{z}_{n}\right)$, we have $y_{n} \in-\varepsilon_{n} e\left(x_{n}\right)+W\left(x_{n}\right)$. In view of the lower semi-continuity of $f$, for any $y \in f(\bar{x}, z)$, $\tilde{y}_{n} \in f\left(x_{n}, \tilde{z}_{n}\right)$ can be chosen to satisfy $\tilde{y}_{n} \rightarrow y$ and $\tilde{y}_{n}+\varepsilon_{n} e\left(x_{n}\right) \in W\left(x_{n}\right)$. By letting $n \rightarrow+\infty$, $y \in W(\bar{x})$ by the closeness of $W$, and so $f(\bar{x}, z) \cap(-\operatorname{int} C(\bar{x}))=\emptyset$. Therefore, $\bar{x} \in \Omega$. (GVQEP) is GTWP1 and also GTWP2.

It is easy to see that the conclusion of Theorem 2.6 still holds if (b1) is replaced by ' $E$ is compact.' In addition, if $f$ is compact-valued, then (b1) can also be substituted by:

(b2) $\phi$ is compact-level on $\Omega_{1}\left(\varepsilon_{0}\right)$ for some $\varepsilon_{0}>0$, or

(b3) $X$ is a finite-dimensional normed linear space and $\phi$ is level-bounded on $E$.

Indeed $\Omega_{1}\left(\varepsilon_{0}\right)=\left\{x \in \Omega_{1}\left(\varepsilon_{0}\right): \phi(x) \leq \varepsilon\right\}$ is compact for each $\varepsilon \geq \varepsilon_{0}$ by (b2) and so (b1) holds. Define $A(\varepsilon)=\{x \in E: \phi(x) \leq \varepsilon\}$ for each $\varepsilon \in \mathbb{R}$. Then $A(\varepsilon)$ is bounded by (b3), otherwise, there exists $\left\{u_{n}\right\} \subset A(\varepsilon) \subset E$ such that $\left\|u_{n}\right\| \rightarrow+\infty$ and $\phi\left(u_{n}\right) \leq \varepsilon$. This is absurd according to (b3). $A(\varepsilon)$ is closed since $\phi$ is lower semi-continuous by Proposition 2.1 and so it is compact. Clearly, $\Omega_{1}(\varepsilon) \subset A(\varepsilon)$. Thus, (b1) is satisfied by Lemma 2.1(i).

In fact, GTWPness1 or GTWPness2 for (GVQEP) can fail without the lower semicontinuity of $f$. The following example only states the fact under the assumption that ' $E$ is compact'.

Example 2.2 Let $X=Y=Z=\mathbb{R}, E=[-1,1], F=[0,1], P(x)=\{x\}, Q(x)=F, C(x)=\mathbb{R}_{+}$, $e(x)=1$ for all $x \in[-1,1]$, and

$$
f(x, z)= \begin{cases}{[x+z, x+z+1]} & \text { if } x \in(0,1], z \in[0,1], \\ {[-2,-1]} & \text { if } x=0, z \in[0,1], \\ {[x+z-1, x+z]} & \text { if } x \in[-1,0), z \in[0,1]\end{cases}
$$

(GVQEP) is to find $\bar{x} \in[-1,1]$ such that

$$
f(\bar{x}, z) \cap(-\infty, 0)=\emptyset \quad \text { for all } z \in[0,1] .
$$

It is easy to know $\Omega=(0,1]$. (GVQEP) is neither GTWP2 nor GTWP1. As a matter of fact, $x_{n} \in \Omega_{1}\left(\varepsilon_{n}\right)$ if $\varepsilon_{n}=\frac{1}{n}$ and $x_{n}=\frac{1}{n}$. Again, taking $\tilde{z}_{n}=0$, we have

$$
f\left(x_{n}, \tilde{z}_{n}\right) \cap\left(\varepsilon_{n}-\mathbb{R}_{+}\right)=\left[\frac{1}{n}, \frac{1}{n}+1\right] \cap\left(-\infty, \frac{1}{n}\right] \neq \emptyset .
$$

Thus $x_{n} \in \Omega_{2}\left(\varepsilon_{n}\right)$, in other words, $\left\{x_{n}\right\}$ is an ASS2 of (GVQEP), but $x_{n} \rightarrow 0 \notin \Omega$. It is worth noting that (a2)-(a5) are satisfied, but $f$ is not lower semi-continuous at $(0,0)$. Indeed, let $\left(x_{n}, z_{n}\right)=\left(\frac{1}{n}, \frac{1}{n}\right) \rightarrow(0,0)$. For $y_{0}=-2 \in f(0,0)$ and for any $y_{n} \in f\left(x_{n}, z_{n}\right)=\left[\frac{2}{n}, \frac{2+n}{n}\right],\left\{y_{n}\right\}$ does not converge to $y_{0}$.

By the argument given in the proof of Theorem 3.6, it is easy to yield the following.

Theorem 2.7 If (a2) and (a4) are substituted by ' $E$ is closed and $P(x)=E$ for all $x \in E$ ' and ' $W$ is closed' in Theorem 2.6, respectively, then the conclusion still holds.

Corollary 2.4 If, further, assume that $\Omega$ is a singleton in Theorem 2.6 (resp., 2.7), then (GVQEP) is TWP1 and also TWP2. 
Furthermore, if $E=F=X=Z$ is a locally convex topological space and $E$ is a closed convex subset and if $f$ is single-valued and $P(x)=Q(x)=E$ for all $x \in X$ in Theorem 2.7, then Theorem 2.7 reduces to Corollary 3.1 in [39].

\section{3 (G)HWPness for parametrically GVQEPs}

In this section, the conceptions of HWPness and GHWPness for parametrically GVQEPs are introduced and their sufficient criteria are proposed. Consider the following parametrically $G V Q E P$ : For any given $p \in \Phi$,

$(\mathrm{GVQEP})_{p} \quad$ to find $\bar{x} \in P(\bar{x})$ such that $h(\bar{x}, z, p) \cap(-\operatorname{int} C(\bar{x}))=\emptyset$ for all $z \in Q(\bar{x})$,

where $h: E \times F \times \Phi \rightarrow 2^{Y}, P: E \rightarrow 2^{E}$ and $Q: E \rightarrow 2^{F}$ are strict set-valued mappings, and $(\Phi, \tilde{d})$ is a Hausdorff metric space (parametric space). $\Omega^{p}$ denotes the solution set of $(\mathrm{GVQEP})_{p}$ for each $p \in \Phi$.

If $E=F=X=Z, Y=\mathbb{R}, P(x)=Q(x)=X, C(x)=\mathbb{R}_{+}$for all $x \in X$ and $h$ is single-valued, then $(\mathrm{GVQEP})_{p}$ reduces to the following parametrical $E P$ :

$(\mathrm{EP})_{p} \quad$ to find $\bar{x} \in X$ such that $h(\bar{x}, z, p) \geq 0$ for all $z \in X$.

Definition 3.1 (GVQEP) $)_{p}$ is said to be generalized Hadamard well-posed (in short, GHWP) at $p_{0} \in \Phi$ if $\Omega^{p_{0}} \neq \emptyset$ and for any $\left\{p_{n}\right\} \subset \Phi$ with $p_{n} \rightarrow p_{0}$ and $x_{n} \in \Omega^{p_{n}}$, there exists a subsequence $\left\{x_{n_{i}}\right\}$ of $\left\{x_{n}\right\}$ such that $x_{n_{i}} \rightarrow \bar{x} \in \Omega^{p_{0}}$; to be Hadamard well-posed (in short, HWP) at $p_{0} \in \Phi$ if it is GHWP at $p_{0} \in \Phi$ and $\Omega^{p_{0}}$ is a singleton.

Remark 3.1 (i) Obviously, (GVQEP) $)_{p}$ is GHWP at $p_{0}$ if and only if $\Omega^{p_{0}}$ is nonempty compact and for any $\left\{p_{n}\right\} \subset \Phi$ with $p_{n} \rightarrow p_{0}$ and any $x_{n} \in \Omega^{p_{n}}, d\left(x_{n}, \Omega^{p_{0}}\right) \rightarrow 0$. (GVQEP) $)_{p}$ is HWP at $p_{0}$ if and only if $\Omega^{p_{0}}=\{\bar{x}\}$ and for any $\left\{p_{n}\right\} \subset \Phi$ with $p_{n} \rightarrow p_{0}$ and any $x_{n} \in \Omega^{p_{n}}$, $x_{n} \rightarrow \bar{x}$.

(ii) If $E=F=X=Z, Y=\mathbb{R}, P(x)=Q(x)=X, C(x)=\mathbb{R}_{+}$for all $x \in X$ and $h$ is single-valued, then the HWPness at $p_{0}$ for $(\mathrm{GVQEP})_{p}$ reduces to the HWPness at $p_{0}$ for $(\mathrm{EP})_{p}$, which was investigated by Bianchi et al. [35].

In general, $\mathrm{HWPness}$ for $(\mathrm{GVQEP})_{p}$ at $p_{0}$ implies that diam $\Omega^{p_{n}} \rightarrow 0$ as $p_{n} \rightarrow p_{0}$, since

$$
\operatorname{diam} \Omega^{p_{n}}=\sup \left\{d(a, b): a, b \in \Omega^{p_{n}}\right\} \leq \sup \left\{(d(a, \bar{x})+d(b, \bar{x})): a, b \in \Omega^{p_{n}}\right\},
$$

where $\Omega^{p_{0}}=\{\bar{x}\}$. However the converse fails to be true. See the following example.

Example 3.1 Let $X=Y=Z=\mathbb{R}, \Phi=E=F=\mathbb{R}_{+}, P(x)=\{x\}, Q(x)=C(x)=\mathbb{R}_{+}$for all $x \in E$ and

$$
h(x, z, p)= \begin{cases}{[-x+z,-x+z+1]} & \text { if } p=0, x \in E, z \in F \\ {\left[-x+z+p+\frac{1}{p},-x+z+2 p+\frac{1}{p}\right]} & \text { if } p>0, x \in\left[\frac{1}{p}, p+\frac{1}{p}\right], z \in F \\ {[-1,0],} & \text { otherwise. }\end{cases}
$$

Then

$$
\Omega^{p}= \begin{cases}\{0\} & \text { if } p=0 \\ {\left[\frac{1}{p}, p+\frac{1}{p}\right]} & \text { if } p>0\end{cases}
$$


Set $p_{0}=0$. diam $\Omega^{p_{n}}=p_{n} \rightarrow 0$ for any $p_{n}>0$ with $p_{n} \rightarrow p_{0}$, but $(\text { GVQEP })_{p}$ is not HWP at $p_{0}$. In fact, by taking $x_{n}=p_{n}+\frac{1}{p_{n}} \in \Omega^{p_{n}}, x_{n} \rightarrow+\infty$.

Theorem 3.1 Let $E$ be compact, $p_{0} \in \Phi$ and $\Omega^{p_{0}} \neq \emptyset$. If (a2)-(a4) and

(a7) $h$ is lower semi-continuous at $\left(x, z, p_{0}\right)$ for each $(x, z) \in E \times F$

hold, then $(G V Q E P)_{p}$ is GHWP at $p_{0}$. If, further, $\Omega^{p_{0}}$ is a singleton, then $(G V Q E P)_{p}$ is $H W P$ at $p_{0}$.

Proof For any $p_{n} \rightarrow p_{0}$ and $x_{n} \in \Omega^{p_{n}}$, we have $x_{n} \in P\left(x_{n}\right)$ and

$$
h\left(x_{n}, z_{n}, p_{n}\right) \cap\left(-\operatorname{int} C\left(x_{n}\right)\right)=\emptyset \quad \text { for all } z_{n} \in Q\left(x_{n}\right),
$$

that is,

$$
y_{n} \in W\left(x_{n}\right)
$$

for all $y_{n} \in h\left(x_{n}, z_{n}, p_{n}\right)$. Without loss of generality, assume that $x_{n} \rightarrow \bar{x} \in E$. For any $z \in$ $Q(\bar{x})$, there exists $\tilde{z}_{n} \in Q\left(x_{n}\right)$ such that $\tilde{z}_{n} \rightarrow z$ by (a3) and for any $y \in h\left(\bar{x}, z, p_{0}\right)$, there exists $\tilde{y}_{n} \in h\left(x_{n}, \tilde{z}_{n}, p_{n}\right)$ such that $\tilde{y}_{n} \rightarrow y$ by (a7). Since both $P$ and $W$ are closed, $\bar{x} \in P(\bar{x})$ and $y \in W(\bar{x})$ by (3.1), and so

$$
h\left(\bar{x}, z, p_{0}\right) \cap(-\operatorname{int} C(\bar{x}))=\emptyset \quad \text { for all } z \in Q(\bar{x}) .
$$

This deduces that $\bar{x} \in \Omega^{p_{0}}$ and $(\mathrm{GVQEP})_{p}$ is GHWP at $p_{0}$. The second conclusion follows directly from Definition 3.1.

The conclusion in Theorem 3.1 is still true if (a2) is replaced by the assumption that $P$ is closed-valued and upper semi-continuous on $E$. Theorem 3.1 can be false without (a7). See the instance as follows.

Example 3.2 Let $X=Y=Z=\mathbb{R}, E=[0,1], \Phi=F=\mathbb{R}_{+}, P(x)=\{x\}, Q(x)=C(x)=\mathbb{R}_{+}$for all $x \in E$, and $h$ is defined by

$$
h(x, z, p)= \begin{cases}{[-x+z,-x+z+1]} & \text { if } p=0, x \in E, z \in F, \\ {\left[-x+z+\frac{1}{p},-x+z+1+\frac{1}{p}\right]} & \text { if } p>0, x \in E, z \in F,\end{cases}
$$

(resp.,

$$
h(x, z, p)=\left\{\begin{array}{ll}
{\left[-x+z+\frac{1}{2},-x+z+1\right]} & \text { if } p=0, x \in E, z \in F, \\
{\left[-x+z+\frac{1}{p},-x+z+1+\frac{1}{p}\right]} & \text { if } p>0, x \in E, z \in F .
\end{array}\right)
$$

$(\text { GVQEP })_{p}$ is not HWP (resp., GHWP) at $p_{0}=0$. It is worth noting that $h$ is not lower semicontinuous at $(0,0,0)$. Indeed, take $\left(x_{n}, z_{n}, p_{n}\right)=\left(\frac{1}{n}, \frac{1}{n}, \frac{1}{n}\right) \rightarrow(0,0,0)$. For $\bar{y}=1 \in h(0,0,0)$ and for any $y_{n} \in h\left(x_{n}, z_{n}, p_{n}\right)=[n, n+1]$, it is impossible that the case $y_{n} \rightarrow \bar{y}$ happens. 


\section{P(G)TWPness for parametrical system of GVQEPs}

The main topic of this section is (G)TWPness for parametrical system of GVQEPs, which is a common extension of both (G)TWPness and (G)HWPness, and its the metric characterizations and sufficient conditions. The parametrical system of GVQEPs is

$$
\left\{(\mathrm{GVQEP})_{p}: p \in \Phi\right\}
$$

where $(\mathrm{GVQEP})_{p}$ for each $p \in \Phi$ is described at the beginning of Section 4 and $(\Phi, \tilde{d})$ is a Hausdorff metric space (parametric space).

For any $p \in \Phi$ and $\varepsilon \geq 0$, we list some conditions as follows:

$$
\begin{aligned}
& d(x, P(x)) \leq \varepsilon, \\
& h(x, z, p) \cap(-\varepsilon e(x)-\operatorname{int} C(x))=\emptyset \quad \text { for all } z \in Q(x), \\
& h(x, \tilde{z}, p) \cap(\varepsilon e(x)-C(x)) \neq \emptyset \text { for some } \tilde{z} \in Q(x),
\end{aligned}
$$

and denote

$$
\begin{aligned}
& \Omega_{1}^{p}(\varepsilon)=\{x \in E: x \text { satisfies (4.1) and (4.2) }\}, \\
& \Omega_{2}^{p}(\varepsilon)=\{x \in E: x \text { satisfies (4.1)-(4.3) }\} .
\end{aligned}
$$

Incidentally, for any given $p \in \Phi$, define $f_{p}: E \times F \rightarrow 2^{Y}$ as

$$
f_{p}(x, z)=h(x, z, p) \quad \text { for all }(x, z) \in E \times F .
$$

Then the GVQEP related to $p \in \Phi$ is

$$
p \text {-(GVQEP) } \quad \text { to find } \bar{x} \in P(\bar{x}) \text { such that } f_{p}(\bar{x}, z) \cap(-\operatorname{int} C(\bar{x}))=\emptyset \text { for all } z \in Q(\bar{x}) \text {. }
$$

Clearly, $\Omega^{p}, \Omega_{1}^{p}(\varepsilon)$ and $\Omega_{2}^{p}(\varepsilon)$ are just the solution set, the type I $\varepsilon$-approximating solution set and the type II $\varepsilon$-approximating solution set of $p$-(GVQEP), respectively, for each $p \in \Phi$ and $\varepsilon \in \mathbb{R}_{+}$.

Definition 4.1 For each $p \in \Phi$ and $\varepsilon, \delta \in \mathbb{R}_{+}$, the type $I(\varepsilon, \delta)$-approximating solution set related to $p \in \Phi$ (resp., type II $(\varepsilon, \delta)$-approximating solution set related to $p \in \Phi)$ of $\left\{(\mathrm{GVQEP})_{p}: p \in \Phi\right\}$ is defined as

$$
\begin{aligned}
& \Omega_{1}^{p}(\varepsilon, \delta)=\bigcup\left\{\Omega_{1}^{q}(\varepsilon): \tilde{d}(q, p) \leq \delta\right\} \\
& \left(\text { resp., } \Omega_{2}^{p}(\varepsilon, \delta)=\bigcup\left\{\Omega_{2}^{q}(\varepsilon): \tilde{d}(q, p) \leq \delta\right\}\right) .
\end{aligned}
$$

A sequence $\left\{x_{n}\right\}$ is called a type I approximating solution sequence related to $p \in \Phi$, $\operatorname{ASS1}(p)$ for brevity (resp., type II approximating solution sequence related to $p \in \Phi$, $\operatorname{ASS} 2(p)$ for brevity) of $\left\{(\mathrm{GVQEP})_{p}: p \in \Phi\right\}$ if there exist $\left\{\varepsilon_{n}\right\},\left\{\delta_{n}\right\} \subset \mathbb{R}_{+}$with $\varepsilon_{n}, \delta_{n} \rightarrow 0$ such that $x_{n} \in \Omega_{1}^{p}\left(\varepsilon_{n}, \delta_{n}\right)$ (resp., $\left.x_{n} \in \Omega_{2}^{p}\left(\varepsilon_{n}, \delta_{n}\right)\right)$. 
Remark 4.1 (i) An ASS2(p) of $\left\{(\mathrm{GVQEP})_{p}: p \in \Phi\right\}$ is its $\operatorname{ASS1}(p)$ for each $p \in \Phi$.

(ii) Apparently,

$$
\Omega^{p} \subset \Omega_{1}^{p}(\varepsilon) \subset \Omega_{1}^{p}(\varepsilon, \delta) \quad \text { and } \quad \Omega_{2}^{p}(\varepsilon) \subset \Omega_{2}^{p}(\varepsilon, \delta)
$$

for each $p \in \Phi$ and $\varepsilon, \delta \in \mathbb{R}_{+}$. Also, for each $p \in \Phi$ and $\varepsilon \in \mathbb{R}_{+}$,

$$
\Omega^{p} \subset \Omega_{2}^{p}(\varepsilon)
$$

on the assumption of

(a8) $0 \in h(x, Q(x), p)$ for all $x \in E$ and $p \in \Phi$.

Definition 4.2 $\left\{(\mathrm{GVQEP})_{p}: p \in \Phi\right\}$ is said to be GTWP1 (resp., GTWP2) if for each $p \in$ $\Phi, \Omega^{p} \neq \emptyset$ and for any $\operatorname{ASS} 1(p)$ (resp., $\left.\operatorname{ASS} 2(p)\right)\left\{x_{n}\right\}$ of $\left\{(\mathrm{GVQEP})_{p}: p \in \Phi\right\}$, there exists a subsequence $\left\{x_{n_{i}}\right\}$ such that $x_{n_{i}} \rightarrow \bar{x} \in \Omega^{p}$; to be TWP1 (resp., TWP2) if it is GTWP1 (resp., GTWP2) and $\Omega^{p}$ is a singleton for each $p \in \Phi$.

Remark 4.2 (i) The GTWPness1 for $\left\{(\mathrm{GVQEP})_{p}: p \in \Phi\right\}$ implies its GTWPness2 according to Remark 4.1(i).

(ii) $\left\{(\mathrm{GVQEP})_{p}: p \in \Phi\right\}$ is GTWP1 if and only if for each $p \in \Phi, \Omega^{p}$ is nonempty compact and for any $\operatorname{ASS} 1(p)\left\{x_{n}\right\}$ for $\left\{(\mathrm{GVQEP})_{p}: p \in \Phi\right\}, d\left(x_{n}, \Omega^{p}\right) \rightarrow 0$. When $\Omega^{p}$ is compact for each $p \in \Phi,\left\{(\mathrm{GVQEP})_{p}: p \in \Phi\right\}$ is GTWP2 if and only if for any $p \in \Phi, \Omega^{p} \neq \emptyset$ and for any $\operatorname{ASS} 2(p)\left\{x_{n}\right\}$ for $\left\{(\mathrm{GVQEP})_{p}: p \in \Phi\right\}, d\left(x_{n}, \Omega^{p}\right) \rightarrow 0$. In addition, $\left\{(\mathrm{GVQEP})_{p}: p \in \Phi\right\}$ is TWP1 (resp., TWP2) if and only if for each $p \in \Phi, \Omega^{p}=\left\{\bar{x}^{p}\right\}$ and for any $\operatorname{ASS1}(p)$ (resp., $\operatorname{ASS} 2(p))\left\{x_{n}\right\}$ for $\left\{(\mathrm{GVQEP})_{p}: p \in \Phi\right\}, d\left(x_{n}, \bar{x}^{p}\right) \rightarrow 0$.

Lemma 4.1 Assume that (a2)-(a5) hold and

(a9) $h$ is lower semi-continuous on $E \times F \times \Phi$;

(a10) $\Phi$ is compact.

Then the following facts are true:

(i) $\Omega_{1}^{p}(\varepsilon, \delta)$ is closed for each $p \in \Phi$ and $\varepsilon, \delta>0$.

(ii) $\Omega^{p}=\bigcap\left\{\Omega_{1}^{p}(\varepsilon, \delta): \varepsilon, \delta>0\right\}$ for each $p \in \Phi$.

Proof (i) Let $\left\{x_{n}\right\} \subset \Omega_{1}^{p}(\varepsilon, \delta)$ with $x_{n} \rightarrow \bar{x}$ for each $\varepsilon, \delta>0$. Then there exists $p_{n} \in \Phi$ with $p_{n} \rightarrow p$ such that $x_{n} \in \Omega_{1}^{p_{n}}(\varepsilon)$. Without loss of generality, $\tilde{d}\left(p_{n}, p\right) \leq \delta$. Thus, $d\left(x_{n}, P\left(x_{n}\right)\right) \leq$ $\varepsilon$ and

$$
h\left(x_{n}, z_{n}, p_{n}\right) \cap\left(-\varepsilon e\left(x_{n}\right)-\operatorname{int} C\left(x_{n}\right)\right)=\emptyset \quad \text { for all } z_{n} \in Q\left(x_{n}\right) .
$$

By (a10), without loss of generality, $p_{n} \rightarrow \bar{p} \in \Phi$, which implies that $\tilde{d}(\bar{p}, p) \leq \delta$. Applying the analogous argument given in the proof of Lemma 2.1(i), we have $d(\bar{x}, P(\bar{x})) \leq \varepsilon$ and

$$
h(\bar{x}, z, \bar{p}) \cap(-\varepsilon e(\bar{x})-\operatorname{int} C(\bar{x}))=\emptyset \quad \text { for all } z \in Q(\bar{x}) .
$$

Accordingly, $\bar{x} \in \Omega_{1}^{\bar{p}}(\varepsilon)$ and so $\bar{x} \in \Omega_{1}^{p}(\varepsilon, \delta)$ by $\tilde{d}(\bar{p}, p) \leq \delta$. 
(ii) Obviously, $\Omega^{p} \subset \bigcap\left\{\Omega_{1}^{p}(\varepsilon, \delta): \varepsilon, \delta>0\right\}$ on account of (4.4). Let $\bar{x} \in \Omega_{1}^{p}(\varepsilon, \delta)$ for all $\varepsilon, \delta>0$. Then there exist $\varepsilon_{n} \downarrow 0$ and $\delta_{n} \downarrow 0$ such that

$$
\bar{x} \in \Omega_{1}^{p}\left(\varepsilon_{n}, \delta_{n}\right)=\left\{\bigcup \Omega_{1}^{q}\left(\varepsilon_{n}\right): \tilde{d}(q, p) \leq \delta_{n}\right\} .
$$

Therefore, there exists $p_{n} \in \Phi$ such that $\tilde{d}\left(p_{n}, p\right) \leq \delta_{n}$ and $\bar{x} \in \Omega_{1}^{p_{n}}\left(\varepsilon_{n}\right)$. As a result, $p_{n} \rightarrow p, \bar{x} \in P(\bar{x})$ and $h\left(\bar{x}, z, p_{n}\right) \cap\left(-\varepsilon_{n} e(\bar{x})-\right.$ int $\left.C(\bar{x})\right)=\emptyset$ for all $z \in Q(\bar{x})$. Then $h(\bar{x}, z, p) \cap$ $(-\operatorname{int} C(\bar{x}))=\emptyset$ for all $z \in Q(\bar{x})$ proceeds from (a2), (a3) and (a10) and so $\bar{x} \in \Omega^{p}$.

By a resemblant argument given in the proof of Lemma 2.2, we have the following.

Lemma 4.2 Let (a2)-(a5) and (a9)-(a10) hold. Then the following assertions hold:

(i) $\Omega_{2}^{p}(\varepsilon, \delta)$ is closed for each $p \in \Phi$ and $\varepsilon, \delta>0$.

(ii) $\Omega^{p}=\bigcap\left\{\Omega_{2}^{p}(\varepsilon, \delta): \varepsilon, \delta>0\right\}$ for each $p \in \Phi$.

Theorem 4.1 Let E be bounded.

(i) If $\left\{(G V Q E P)_{p}: p \in \Phi\right\}$ is GTWP1, then for each $p \in \Phi$,

$$
\Omega_{1}^{p}(\varepsilon, \delta) \neq \emptyset \quad \text { for all } \varepsilon, \delta>0 \text { and } \lim _{(\varepsilon, \delta) \rightarrow(0,0)} \alpha\left(\Omega_{1}^{p}(\varepsilon, \delta)\right)=0
$$

(ii) Suppose that (a2)-(a5) and (a9)-(a10) hold. If (4.6) holds for each $p \in \Phi$, then $\left\{(G V Q E P)_{p}: p \in \Phi\right\}$ is GTWP1.

Proof (i) Since $\left\{(\mathrm{GVQEP})_{p}: p \in \Phi\right\}$ is GTWP1, $\Omega^{p}$ is nonempty compact for each $p \in \Phi$. Then $\Omega_{1}^{p}(\varepsilon, \delta) \neq \emptyset$ and

$$
\alpha\left(\Omega_{1}^{p}(\varepsilon, \delta)\right) \leq 2 H\left(\Omega_{1}^{p}(\varepsilon, \delta), \Omega^{p}\right)+\alpha\left(\Omega^{p}\right)=2 \tilde{e}\left(\Omega_{1}^{p}(\varepsilon, \delta), \Omega^{p}\right)
$$

for any $p \in \Phi$ and $\varepsilon, \delta>0$. It is enough to testify $\tilde{e}\left(\Omega_{1}^{p}(\varepsilon, \delta), \Omega^{p}\right) \rightarrow 0$ as $(\varepsilon, \delta) \rightarrow(0,0)$ for each $p \in \Phi$. Otherwise, there exist $p, p_{n} \in \Phi, r>0, \varepsilon_{n} \downarrow 0$ and $\delta_{n} \downarrow 0$ with $\tilde{d}\left(p_{n}, p\right) \leq \delta_{n}$ and $x_{n} \in \Omega_{1}^{p_{n}}\left(\varepsilon_{n}\right)$ such that $d\left(x_{n}, \Omega^{p}\right) \geq r$. This says that $\left\{x_{n}\right\}$ is an $\operatorname{ASS} 1(p)$ of $\left\{(\mathrm{GVQEP})_{p}: p \in\right.$ $\Phi\}$. By Remark 4.2(ii), $d\left(x_{n}, \Omega^{p}\right) \rightarrow 0$, which contradicts $d\left(x_{n}, \Omega^{p}\right) \geq r$.

(ii) For any $\operatorname{ASS} 1(p)\left\{x_{n}\right\}$ of $\left\{(\mathrm{GVQEP})_{p}: p \in \Phi\right\}$, there exist $\left\{\varepsilon_{n}\right\},\left\{\delta_{n}\right\} \subset \mathbb{R}_{+}$with $\left(\varepsilon_{n}, \delta_{n}\right) \rightarrow(0,0)$ such that $x_{n} \in \Omega_{1}^{p}\left(\varepsilon_{n}, \delta_{n}\right)$ by Remark 4.2(ii). In view of Lemma 4.1 and Kuratowski theorem [51], $\Omega^{p}$ is nonempty compact and $H\left(\Omega_{1}^{p}\left(\varepsilon_{n}, \delta_{n}\right), \Omega^{p}\right) \rightarrow 0$. Thus $d\left(x_{n}, \Omega^{p}\right) \rightarrow 0$. It follows that $\left\{(\mathrm{GVQEP})_{p}: p \in \Phi\right\}$ is GTWP1.

\section{Theorem 4.2 Let $E$ be bounded.}

(i) Suppose that for each $p \in \Phi, \Omega^{p}$ is compact. If $\left\{(G V Q E P)_{p}: p \in \Phi\right\}$ is GTWP2, then for $p \in \Phi$,

$$
\Omega_{2}^{p}(\varepsilon, \delta) \neq \emptyset \quad \text { for all } \varepsilon, \delta>0, \text { and } \lim _{(\varepsilon, \delta) \rightarrow(0,0)} \alpha\left(\Omega_{2}^{p}(\varepsilon, \delta)\right)=0
$$

(ii) Assume that (a2)-(a5) and (a8)-(a10) hold. If (4.7) holds for each $p \in \Phi$, then $\left\{(G V Q E P)_{p}: p \in \Phi\right\}$ is GTWP2. 
Proof This proof is completed by using Lemma 4.2 and a similar argument proposed in the proof of Theorem 2.2 and is omitted.

Corollary 4.1 Assume that $E$ is bounded and $\Omega^{p}$ is a singleton for each $p \in \Phi$.

(i) If $\left\{(G V Q E P)_{p}: p \in \Phi\right\}$ is TWP1 (resp., TWP2), then (4.6) (resp., (4.7)) holds for each $p \in \Phi$.

(ii) Assume that (a2)-(a5) and (a9)-(a10) (resp., (a2)-(a5) and (a8)-(a10)) hold. If (4.6) (resp., (4.7)) holds for each $p \in \Phi$, then $\left\{(G V Q E P)_{p}: p \in \Phi\right\}$ is TWP1 (resp., TWP2).

By a similar method of the proof in Theorem 2.6, we have the following.

Theorem 4.3 Let (a2)-(a5) and (a9)-(a10) hold and $\Omega^{p} \neq \emptyset$ for each $p \in \Phi$. If

(b4) For each $p \in \Phi, \Omega_{1}^{p}\left(\varepsilon_{0}, \delta_{0}\right)$ is compact for some $\varepsilon_{0}, \delta_{0}>0$; or

(b5) $X$ is a finite-dimensional normed linear space and for each $p \in \Phi, \Omega_{1}^{p}\left(\varepsilon_{0}, \delta_{0}\right)$ is bounded for some $\varepsilon_{0}, \delta_{0}>0$

holds, then $\left\{(G V Q E P)_{p}: p \in \Phi\right\}$ is GTWP1 and also GTWP2.

Corollary 4.2 Further suppose that $\Omega^{p}$ is a singleton for each $p \in \Phi$ in Theorem 4.3. Then $\left\{(G V Q E P)_{p}: p \in \Phi\right\}$ is TWP1 and also TWP2.

\section{Relations among the types of proposed well-posedness}

In this section we are interested in the comparison among the types of proposed wellposedness defined in previous sections.

It seems on the surface to have no relations between the (G)HWPness for (GVQEP) and (G)TWPness for (GVQEP). However, if there are some connections between their objective mappings, we may discuss the relations.

Example 5.1 Let $\Phi=\mathbb{R}_{+}$, and let $h: E \times F \times \Phi \rightarrow 2^{Y}$ be the objective mapping of $(\mathrm{GVQEP})_{p}$ and $f: E \times F \rightarrow 2^{Y}$ be the objective mapping of (GVQEP), where

$$
h(x, z, p)=f(x, z)+p e(x) \quad \text { for all }(x, z, p) \in E \times F \times \Phi .
$$

And let $\Omega$ be the solution set of (GVQEP) and $\Omega_{1}(p)$ (resp., $\Omega_{2}(p)$ ) be the type I $p$-approximating solution set (resp., type II $p$-approximating solution set) of (GVQEP). It follows that

$$
\Omega^{p} \subset \Omega_{1}(p)
$$

and

$$
\Omega^{p} \subset \Omega_{2}(p)
$$

if (a6) holds. If, further, $P(x)=E$ for all $x \in E$, then both (5.2) and (5.3) are indeed equalities.

Figure 1 illuminates the relations among GHWPness for (GVQEP) $)_{p}$, GTWPness1 and GTWPness2 for (GVQEP) when the relation of their objective mapping is defined by (5.1). If, further, $\Omega=\Omega^{0}$ is a singleton, then Figure 1 illuminates the relations among HWPness for (GVQEP) $)_{p}$, TWPness1 and TWPness2 for (GVQEP) when the relation of their objective mapping is defined by (5.1). 


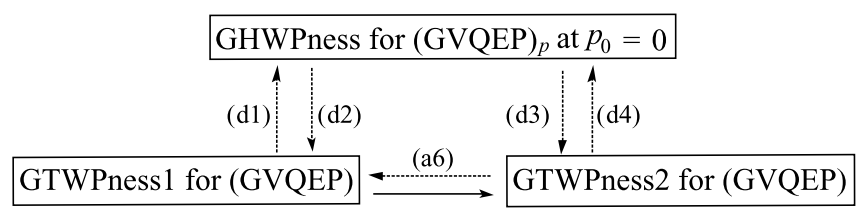

(d1) The connections between two objective mappings are defined as (5.1);

(d2) $P(x)=E$ for all $x \quad E$ and (d1) holds;

(d3) Both (d1) and (a6) hold;

(d4) Both (d2) and (a6) hold.

Figure 1 The relation between GTWPness for (GVQEP) and GHWPness for (GVQEP) $)_{p}$ at $p_{0}=0$.

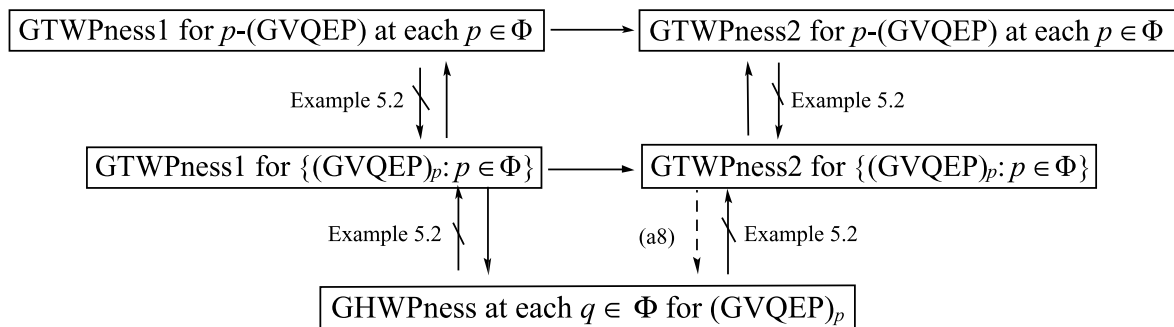

Figure 2 The relations between GTWPness for $p$-(GVQEP) and GTWPness for $\left\{(\text { GVQEP })_{p}\right\}$ and between GHWPness for (GVQEP) $)_{p}$ and GTWPness for $\left\{(\mathrm{GVQEP})_{p}: p \in \Phi\right\}$.

It follows from (4.4) and (4.5) that GTWPness1 and GTWPness2 for $\left\{(\mathrm{GVQEP})_{p}: p \in\right.$ $\Phi$ \} imply that GTWPness1 and GTWPness2 for $p$-(GVQEP) for each $p \in \Phi$, respectively, and GTWPness1 for $\left\{(\mathrm{GVQEP})_{p}: p \in \Phi\right\}$ also implies GHWPness for $(\mathrm{GVQEP})_{p}$ at each $p \in \Phi$, while GTWPness2 implies GHWPness for (GVQEP) ${ }_{p}$ at each $p \in \Phi$ if (a10) holds. But these converses fail to hold. See the following example.

Example 5.2 Let $X=Y=Z=\mathbb{R}, \Phi=E=F=\mathbb{R}_{+}, P(x)=\{x\}, Q(x)=C(x)=\mathbb{R}_{+}, e(x)=1$ for all $x \in E$ and $h$ defined by

$$
h(x, z, p)= \begin{cases}{[-x+z+1,-x+z+2]} & \text { if } p=0, x \in E, z \in F \\ {[-p x+z+p,-p x+z+2 p]} & \text { if } p>0, x \in E, z \in F .\end{cases}
$$

Then $\Omega^{p}=[0,1]$,

$$
\Omega_{1}^{p}(\varepsilon)=\left\{\begin{array}{ll}
{[0,1+\varepsilon],} & \text { if } p=0, \\
{\left[0,1+\frac{\varepsilon}{p}\right],} & \text { if } p>0,
\end{array} \quad \Omega_{2}^{p}(\varepsilon)= \begin{cases}{[1-\varepsilon, 1+\varepsilon]} & \text { if } p=0, \\
{\left[1-\frac{\varepsilon}{p}, 1+\frac{\varepsilon}{p}\right]} & \text { if } p \geq \varepsilon \\
{\left[0,1+\frac{\varepsilon}{p}\right],} & \text { otherwise }\end{cases}\right.
$$

and

$$
\Omega_{1}^{0}(\varepsilon, \delta)=\Omega_{2}^{0}(\varepsilon, \delta)=[0,+\infty),
$$

for each $p \in \Phi$ and $0<\varepsilon, \delta<1$. It is clear that (GVQEP) $)_{p}$ is GHWP at each $p \in \Phi$, and $p$-(GVQEP) is GTWP1 and GTWP2 for each $p \in \Phi$, while $\left\{(\mathrm{GVQEP})_{p}: p \in \Phi\right\}$ is neither 
GTWP1 nor GTWP2. In fact, take $p_{n}=\frac{1}{n^{2}}, \varepsilon_{n}=\frac{1}{n}, \delta_{n}=\frac{1}{n^{2}}$ and $x_{n}=n$. It is easy to see that $\left\{x_{n}\right\}$ is an $\operatorname{ASS} 1(0)$ (resp., $\left.\operatorname{ASS} 2(0)\right)$ of $\left\{(\mathrm{GVQEP})_{p}: p \in \Phi\right\}$, but it has no convergent subsequence.

Figure 2 illuminates the relation between GTWPness for $\left\{(\mathrm{GVQEP})_{p}: p \in \Phi\right\}$ and GTWPness for $p$-(GVQEP) for each $p \in \Phi$, and that between GTWPness for $\left\{(\mathrm{GVQEP})_{p}: p \in\right.$ $\Phi\}$ and GHWPness for (GVQEP) $p$ at each $p \in \Phi$.

\section{Competing interests}

The authors declare that they have no competing interests.

\section{Authors' contributions}

All authors contributed equally and significantly in writing this paper. All authors read and approved the final manuscript.

\section{Acknowledgements}

This research is supported by the Doctoral Fund of innovation of Beijing University of Technology.

Received: 23 September 2013 Accepted: 5 December 2013 Published: 07 Jan 2014

\section{References}

1. Tykhonov, AN: On the stability of the functional optimization problem. USSR Comput. Math. Math. Phys. 6, 28-33 (1966)

2. Levitin, ES, Polyak, BT: Convergence of minimizing sequences in conditional extremum problems. Sov. Math. Dokl. 7, 764-767 (1966)

3. Dontchev, AL, Zolezzi, T: Well-Posed Optimization Problems. Lecture Notes in Mathematics, vol. 1543. Springer, Berlin (1993)

4. Zaslavski, AJ: Optimization on Metric and Normed Spaces. Springer Optimization and Its Applications. Springer, New York (2010)

5. Zaslavski, AJ: Nonconvex Optimal Control and Variational Problems. Springer Optimization and Its Applications. Springer, New York (2013)

6. Papalia, M: On well-posedness in vector optimization. Università degli studi di Bergamo, Bergamo, Italy (2010)

7. Reich, S, Zaslavski, AJ: A note on well-posed null and fixed point problems. Fixed Point Theory Appl. 2005, 207-211 (2005)

8. Chifu, C, Petruşl, G: Well-posedness and fractals via fixed point theory. Fixed Point Theory Appl. 2008, Article ID 645419 (2008)

9. Petruşel, A, Rus, IA, Yao, JC: Well-posedness in the generalized sense of the fixed-point problems for multivalued operators. Taiwan. J. Math. 3(11), 903-914 (2007)

10. Lemaire, B, Ould Ahmed Salem, C, Revalski, JP: Well-posedness by perturbations of variational problems. J. Optim. Theory Appl. 2(115), 345-368 (2002)

11. Fang, YP, Huang, NJ, Yao, JY: Well-posedness of mixed variational inequalities, inclusion problems and fixed point problems. J. Glob. Optim. 41, 117-133 (2008)

12. Ceng, LC, Yao, JC: Well-posedness of generalized mixed variational inequalities, inclusion problems and fixed point problems. Nonlinear Anal., Theory Methods Appl. 69, 4585-4603 (2008)

13. Yu, J, Yang, H, Yu, C: Well-posed Ky Fan's point, quasi-variational inequality and Nash equilibrium problems. Nonlinear Anal., Theory Methods Appl. 66(4), 777-790 (2007)

14. Lignola, MB: Well-posedness and L-well-posedness for quasivariational inequalities. J. Optim. Theory Appl. 1(128), 119-138 (2006)

15. Ceng, LC, Hadjisavvas, N, Schaible, S, Yao, JC: Well-posedness of mixed quasivariational-like inequalities. J. Optim. Theory Appl. 139, 109-125 (2008)

16. Huang, XX, Yang, YQ, Zhu, DC: Levitin-Polyak well-posedness of variational inequalities problems with functional constraints. J. Glob. Optim. 44, 159-174 (2009)

17. Jiang, B, Zhang, J, Huang, XX: Levitin-Polyak well-posedness of generalized quasivariational inequalities with functional constraints. Nonlinear Anal., Theory Methods Appl. 70, 1492-1530 (2009)

18. Zhang, J, Jiang, B, Huang, XX: Levitin-Polyak well-posedness in vector quasivariational inequality problems with functional constraints. Fixed Point Theory Appl. 2010, Article ID 984074 (2010)

19. Xu, Z, Zhu, DC, Huang, XX: Levitin-Polyak well-posedness in generalized vector quasivariational inequality problems with functional constraints. Math. Methods Oper. Res. 3(67), 505-524 (2008)

20. Wang, SH, Huang, NJ: Levitin-Polyak well-posedness for generalized quasi-variational inclusion and disclusion problems and optimization problems with constraints. Taiwan. J. Math. 1(16), 237-257 (2012)

21. Wang, SH, Huang, NJ, Wong, MM: Strong Levitin-Polyak well-posedness for generalized quasi-variational inclusion problems with applications. Taiwan. J. Math. 2(16), 665-690 (2012)

22. Lin, LJ, Chuang, CS: Well-posedness in the generalized sense for variational inclusion and disclusion problems and well-posedness for optimization problems with constraint. Nonlinear Anal., Theory Methods Appl. 70, 3609-3617 (2009)

23. Heemels, WPMH, Schumacher, JM, Weiland, S: Well-posedness of linear complementarity systems. In: Decision and Control, vol. 3, pp. 3037-3042. Proceedings of the 38th IEEE Conference, Phoenix, Arizona, USA (1999)

24. Heemels, PMH, Camlibel, MKC, Van der Schaft, AJ, Schumacher, JM: Well-posedness of the complementarity class of hybrid systems. In: Proc. IFAC 15th Triennial World Congress, Barcelona, Spain (2002) 
25. Margiocco, M, Patrone, F, Chicco, LP: A new approach to Tikhonov well-posedness for Nash equilibria. Optimization 4(40), 385-400 (1997)

26. Margiocco, M, Patrone, F, Chicco, LP: Metric characterizations of Tikhonov well-posedness in value. J. Optim. Theory Appl. 2(100), 377-387 (1999)

27. Lignola, MB, Morgan, J: $\alpha$-Well-posedness for Nash equilibria and for optimization problems with Nash equilibrium constraints. J. Glob. Optim. 3(36), 439-459 (2006)

28. Margiocco, M, Patrone, F, Chicco, LP: On the Tikhonov well-posedness of concave games and Cournot oligopoly games. J. Optim. Theory Appl. 2(112), 361-379 (2002)

29. Morgan, J: Approximations and well-posedness in multicriteria games. Ann. Oper. Res. 137, 257-268 (2005)

30. Scalzo, W: Hadamard well-posedness in discontinuous non-cooperative games. J. Math. Anal. Appl. 360, 697-703 (2009)

31. Peng, JW, Wu, SY: The well-posedness for multiobjective generalized games. J. Optim. Theory Appl. 150, 416-423 (2011)

32. Blum, E, Oettli, W: From optimization and variational inequalities to equilibrium problems. Math. Stud. 63, 123-145 (1994)

33. Long, XJ, Huang, NJ, Teo, KL: Levitin-Polyak well-posedness for equilibrium problems with functional constraints. J. Inequal. Appl. 2008, Article ID 657329 (2008)

34. Zaslavski, AJ: Generic well-posedness for a class of equilibrium problems. J. Inequal. Appl. 2008, Article ID 581917 (2008)

35. Bianchi, M, Kassay, G, Pini, R: Well-posed equilibrium problems. Nonlinear Anal., Theory Methods Appl. 72, 460-468 (2010)

36. Fang, YP, Hu, R: Parametric well-posedness for variational inequalities defined by bifunctions. Comput. Math. Appl. 53, 1306-1316 (2007)

37. Wang, HJ, Cheng, CZ: Parametic well-posedness for quasivariational-like inequalities. Far East J. Math. Sci. 55, 31-47 (2011)

38. Fang, YP, Hu, R, Huang, NJ: Well-posedness for equilibrium problems and for optimization problems with equilibrium constraints. Comput. Math. Appl. 1(55), 89-100 (2008)

39. Li, SJ, Li, MH: Levitin-Polyak well-posedness of vector equilibrium problems. Math. Methods Oper. Res. 69, 125-140 (2009)

40. Peng, JW, Wang, Y, Zhao, LJ: Generalized Levitin-Polyak well-posedness of vector equilibrium problems. Fixed Point Theory Appl. 2009, Article ID 684304 (2009)

41. Zhang, WY: Well-posedness for convex symmetric vector quasi-equilibrium problems. J. Math. Anal. Appl. 387, 909-915 (2012)

42. Wang, G, Huang, XX, Zhang, J, Chen, GY: Levitin-Polyak well-posedness of generalized vector equilibrium problems with functional constraints. Acta Math. Sci. 30(5), 1400-1412 (2010)

43. Peng, JW, Wang, Y, Wu, SY: Levitin-Polyak well-posedness for vector quasi-equilibrium problems with functional constraints. Taiwan. J. Math. 2(16), 635-649 (2012)

44. Peng, JW, Wang, Y, Wu, SY: Levitin-Polyak well-posedness of generalized vector equilibrium problems. Taiwan. J. Math 5(15), 2311-2330 (2011)

45. Peng, JW, Wang, Y, Wu, SY: Levitin-Polyak well-posedness of generalized vector quasi-equilibrium problems with functional constraints. J. Glob. Optim. 52, 779-795 (2012)

46. Bianchi, M, Kassay, G, Pini, P: Well-posedness for vector equilibrium problems. Math. Methods Oper. Res. 70, 171-182 (2009)

47. Peng, JW, Wu, SY: The generalized Tykhonov well-posedness for system of vector quasi-equilibrium problems. Optim. Lett. 4, 501-512 (2010)

48. Li, QY, Wang, SH: Well-posedness for parametric strong vector quasi-equilibrium problems with applications. Fixed Point Theory Appl. 2011, Article ID 62 (2011)

49. Aubin, JP, Ekeland, I: Applied Nonlinear Analysis. Wiley, New York (1984)

50. Aubin, JP, Cellina, A: Differential Inclusion. Springer, Berlin (1994)

51. Kuratowski, C: Topologie, vol. 1. Panstwowe Wydawnictwo Naukove, Warsaw (1952)

52. Luc, DT: Theory of Vector Optimization. Lecture Notes in Economics and Mathematical Systems, vol. 319. Springer Berlin (1989)

53. Sach, PH, Tuan, LA: New scalarizing approach to the stability analysis in parametric generalized Ky Fan inequality problems. J. Optim. Theory Appl. 157, 347-364 (2013)

54. Qu, DN, Cheng, CZ: Existence of solutions for generalized vector quasi-equilibrium problems by scalarization method with applications. Abstr. Appl. Anal. 2013, Article ID 916089 (2013)

55. Chen, GY, Yang, XQ, Yu, H: A nonlinear scalarization function and generalized vector quasi-equilibrium problems. J. Glob. Optim. 32, 451-466 (2005)

10.1186/1687-1812-2014-8

Cite this article as: Qu and Cheng: Several types of well-posedness for generalized vector quasi-equilibrium

problems with their relations. Fixed Point Theory and Applications 2014, 2014:8 